\title{
POLARIZATION OPTIMALITY OF EQUALLY SPACED POINTS ON THE CIRCLE FOR DISCRETE POTENTIALS
}

\author{
DOUGLAS P. HARDIN, AMOS P. KENDALL, AND EDWARD B. SAFF
}

\begin{abstract}
We prove a conjecture of Ambrus, Ball and Erdélyi that equally spaced points maximize the minimum of discrete potentials on the unit circle whenever the potential is of the form

$$
\sum_{k=1}^{n} f\left(d\left(z, z_{k}\right)\right)
$$

where $f:[0, \pi] \rightarrow[0, \infty]$ is non-increasing and convex and $d(z, w)$ denotes the geodesic distance between $z$ and $w$ on the circle.
\end{abstract}

\section{Introduction And Main Results}

Let $\mathbb{S}^{1}:=\left\{z=x+i y \mid x, y \in \mathbb{R}, x^{2}+y^{2}=1\right\}$ denote the unit circle in the complex plane $\mathbb{C}$. For $z, w \in \mathbb{S}^{1}$, we denote by $d(z, w)$ the geodesic (shortest arclength) distance between $z$ and $w$. Let $f:[0, \pi] \rightarrow[0, \infty]$ be non-increasing and convex on $(0, \pi]$ with $f(0)=\lim _{\theta \rightarrow 0^{+}} f(\theta)$. It then follows that $f$ is a continuous extended real-valued function on $[0, \pi]$. For a list of $n$ points (not necessarily distinct) $\omega_{n}=\left(z_{1}, \ldots, z_{n}\right) \in\left(\mathbb{S}^{1}\right)^{n}$, we consider the $f$-potential of $\omega_{n}$,

$$
U^{f}\left(\omega_{n} ; z\right):=\sum_{k=1}^{n} f\left(d\left(z, z_{k}\right)\right) \quad\left(z \in \mathbb{S}^{1}\right)
$$

and the $f$-polarization of $\omega_{n}$,

$$
M^{f}\left(\omega_{n} ; \mathbb{S}^{1}\right):=\min _{z \in \mathbb{S}^{1}} U^{f}\left(\omega_{n} ; z\right) .
$$

In this note, we are chiefly concerned with the $n$-point $f$-polarization of $\mathbb{S}^{1}$ (also called the $n$th $f$-Chebyshev constant of $\mathbb{S}^{1}$ ),

$$
M_{n}^{f}\left(\mathbb{S}^{1}\right):=\sup _{\omega_{n} \in\left(\mathbb{S}^{1}\right)^{n}} M^{f}\left(\omega_{n} ; \mathbb{S}^{1}\right),
$$

which has been the subject of several recent papers (e.g., [1], [2], [5], [6]).

Date: August 25, 2012.

2000 Mathematics Subject Classification. Primary 52A40, 30C15.

Key words and phrases. Polarization, Chebyshev constants, roots of unity, potentials, max-min problems.

This research was supported, in part, by the U. S. National Science Foundation under grants DMS-0808093 and DMS-1109266. 
In the case (relating to Euclidean distance) when

$$
f(\theta)=f_{s}(\theta):=\left|e^{i \theta}-1\right|^{-s}=(2 \sin |\theta / 2|)^{-s}, s>0,
$$

we abbreviate the notation for the above quantities by writing

$$
\begin{aligned}
U^{s}\left(\omega_{n} ; z\right) & :=\sum_{k=1}^{n} f_{s}\left(d\left(z, z_{k}\right)\right)=\sum_{k=1}^{n} \frac{1}{\left|z-z_{k}\right|^{s}} \\
M^{s}\left(\omega_{n} ; \mathbb{S}^{1}\right) & :=\min _{z \in \mathbb{S}^{1}} \sum_{k=1}^{n} \frac{1}{\left|z-z_{k}\right|^{s}}, \\
M_{n}^{s}\left(\mathbb{S}^{1}\right) & :=\sup _{\omega_{n} \in\left(\mathbb{S}^{1}\right)^{n}} M^{s}\left(\omega_{n} ; \mathbb{S}^{1}\right) .
\end{aligned}
$$

The main result of this note is the following theorem conjectured by G. Ambrus et al [2]. Its proof is given in the next section.

Theorem 1. Let $f:[0, \pi] \rightarrow[0, \infty]$ be non-increasing and convex on $(0, \pi]$ with $f(0)=\lim _{\theta \rightarrow 0^{+}} f(\theta)$. If $\omega_{n}$ is any configuration of $n$ distinct equally spaced points on $\mathbb{S}^{1}$, then $M^{f}\left(\omega_{n} ; \mathbb{S}^{1}\right)=M_{n}^{f}\left(\mathbb{S}^{1}\right)$. Moreover, if the convexity condition is replaced by strict convexity, then such configurations are the only ones that achieve this equality.

Applying this theorem to the case of $f_{s}$ given in (4) we immediately obtain the following.

Corollary 2. Let $s>0$ and $\omega_{n}^{*}:=\left\{e^{i 2 \pi k / n}: k=1,2, \ldots, n\right\}$. If $\left(z_{1}, \ldots, z_{n}\right) \in$ $\left(\mathbb{S}^{1}\right)^{n}$, then

$$
\min _{z \in \mathbb{S}^{1}} \sum_{k=1}^{n} \frac{1}{\left|z-z_{k}\right|^{s}} \leq M^{s}\left(\omega_{n}^{*} ; \mathbb{S}^{1}\right)=M_{n}^{s}\left(\mathbb{S}^{1}\right)
$$

with equality if and only if $\left(z_{1}, \ldots, z_{n}\right)$ consists of distinct equally spaced points.

The following representation of $M^{s}\left(\omega_{n}^{*} ; \mathbb{S}^{1}\right)$ in terms of Riesz s-energy was observed in [2]:

$$
M^{s}\left(\omega_{n}^{*} ; \mathbb{S}^{1}\right)=\frac{\mathcal{E}_{s}\left(\mathbb{S}^{1} ; 2 n\right)}{2 n}-\frac{\mathcal{E}_{s}\left(\mathbb{S}^{1} ; n\right)}{n},
$$

where

$$
\mathcal{E}_{s}\left(\mathbb{S}^{1} ; n\right):=\inf _{\omega_{n} \in\left(\mathbb{S}^{1}\right)^{n}} \sum_{j=1}^{n} \sum_{\substack{k=1 \\ k \neq j}}^{n} \frac{1}{\left|z_{j}-z_{k}\right|^{s}}
$$


Thus, applying the asymptotic formulas for $\mathcal{E}_{s}\left(\mathbb{S}^{1} ; n\right)$ given in [3], we obtain the dominant term of $M_{n}^{s}\left(\mathbb{S}^{1}\right)$ as $n \rightarrow \infty$ :

$$
M_{n}^{s}\left(\mathbb{S}^{1}\right) \sim\left\{\begin{array}{l}
\frac{2 \zeta(s)}{(2 \pi)^{s}}\left(2^{s}-1\right) n^{s}, \quad s>1, \\
(1 / \pi) n \log n, \quad s=1, \\
\frac{2^{-s}}{\sqrt{\pi}} \frac{\Gamma\left(\frac{1-s}{2}\right)}{\Gamma\left(1-\frac{s}{2}\right)} n, \quad s \in[0,1),
\end{array}\right.
$$

where $\zeta(s)$ denotes the classical Riemann zeta function and $a_{n} \sim b_{n}$ means that $\lim _{n \rightarrow \infty} a_{n} / b_{n}=1$. These asymptotics, but for $M^{s}\left(\omega_{n}^{*} ; \mathbb{S}^{1}\right)$, were stated in $[2]$.

For $s$ an even integer, say $s=2 m$, the precise value of $M_{n}^{2 m}\left(\mathbb{S}^{1}\right)=$ $M^{2 m}\left(\omega_{n}^{*} ; \mathbb{S}^{1}\right)$ can be expressed in finite terms, as can be seen from formula (1.20) in [3].

Corollary 3. We have

$$
M_{n}^{2 m}\left(\mathbb{S}^{1}\right)=\frac{2}{(2 \pi)^{2 m}} \sum_{k=1}^{m} n^{2 k} \zeta(2 k) \alpha_{m-k}(2 m)\left(2^{2 k}-1\right), \quad m \in \mathbb{N},
$$

where $\alpha_{j}(s)$ is defined via the power series for $\operatorname{sinc} z=(\sin \pi z) /(\pi z)$ :

$$
(\operatorname{sinc} z)^{-s}=\sum_{j=0}^{\infty} \alpha_{j}(s) z^{2 j}, \quad \alpha_{0}(s)=1 .
$$

In particular,

$$
\begin{aligned}
M_{n}^{2}\left(\mathbb{S}^{1}\right) & =\frac{2}{(2 \pi)^{2}} n^{2} \zeta(2)=\frac{n^{2}}{4}, \\
M_{n}^{4}\left(\mathbb{S}^{1}\right) & =\frac{2}{(2 \pi)^{4}}\left[n^{2} \zeta(2) \alpha_{1}(4)\left(2^{2}-1\right)+n^{4} \zeta(4)\left(2^{4}-1\right)\right]=\frac{n^{2}}{24}+\frac{n^{4}}{48}, \\
M_{n}^{6}\left(\mathbb{S}^{1}\right) & =\frac{2}{(2 \pi)^{6}}\left[n^{2} \zeta(2) \alpha_{2}(6)\left(2^{2}-1\right)+n^{4} \zeta(4) \alpha_{1}(6)\left(2^{4}-1\right)+n^{6} \zeta(6)\left(2^{6}-1\right)\right] \\
& =\frac{n^{2}}{120}+\frac{n^{4}}{192}+\frac{n^{6}}{480},
\end{aligned}
$$

The case $s=2$ of the above corollary was first proved in [1], 2] and the case $s=4$ was first proved in [5]. We remark that an alternative formula for $\alpha_{j}(s)$ is

$$
\alpha_{j}(s)=\frac{(-1)^{j} B_{2 j}^{(s)}(s / 2)}{(2 j) !}(2 \pi)^{2 j}, \quad j=0,1,2, \ldots,
$$

\footnotetext{
${ }^{1}$ We remark that there is a factor of $2 /(2 \pi)^{p}$ missing in the asymptotics given in [2] for the case $p:=s>1$.
} 
where $B_{j}^{(\alpha)}(x)$ denotes the generalized Bernoulli polynomial. Asymptotic formulas for $M_{n}^{f}\left(\mathbb{S}^{1}\right)$ for certain other functions $f$ can be obtained from the asymptotic formulas given in [4].

As other consequences of Theorem 1, we immediately deduce that equally spaced points are optimal for the following problems

$$
\min _{\omega_{n} \in\left(\mathbb{S}^{1}\right)^{n}} \max _{z \in \mathbb{S}^{1}} \sum_{k=1}^{n}\left|z-z_{k}\right|^{\alpha}, \quad(0<\alpha \leq 1),
$$

and

$$
\max _{\omega_{n} \in\left(\mathbb{S}^{1}\right)^{n}} \min _{z \in \mathbb{S}^{1}} \sum_{k=1}^{n} \log \frac{1}{\left|z-z_{k}\right|},
$$

with the solution to (8) being well-known. Furthermore, various generalizations of the polarization problem for Riesz potentials for configurations on $\mathbb{S}^{1}$ are worthy of consideration, such as minimizing the potential on circles concentric with $\mathbb{S}^{1}$.

\section{Proof of Theorem 1}

For distinct points $z_{1}, z_{2} \in \mathbb{S}^{1}$, we let $\widehat{z_{1} z_{2}}$ denote the closed subarc of $\mathbb{S}^{1}$ from $z_{1}$ to $z_{2}$ traversed in the counterclockwise direction. We further let $\gamma\left(\widehat{z_{1} z_{2}}\right)$ denote the length of $\widehat{z_{1} z_{2}}$ (thus, $\gamma\left(\widehat{z_{1} z_{2}}\right)$ equals either $d\left(z_{1}, z_{2}\right)$ or $\left.2 \pi-d\left(z_{1}, z_{2}\right)\right)$. Observe that the points $z_{1}$ and $z_{2}$ partition $\mathbb{S}^{1}$ into two subarcs: $\widehat{z_{1} z_{2}}$ and $\widehat{z_{2} z_{1}}$. The following lemma (see proof of Lemma 1 in [2]) is a simple consequence of the convexity and monotonicity of the function $f$ and is used to show that any $n$-point configuration $\omega_{n} \subset \mathbb{S}^{1}$ such that $M^{f}\left(\omega_{n} ; \mathbb{S}^{1}\right)=M_{n}^{f}\left(\mathbb{S}^{1}\right)$ must have the property that any local minimum of $U^{f}\left(\omega_{n} ; \cdot\right)$ is a global minimum of this function.

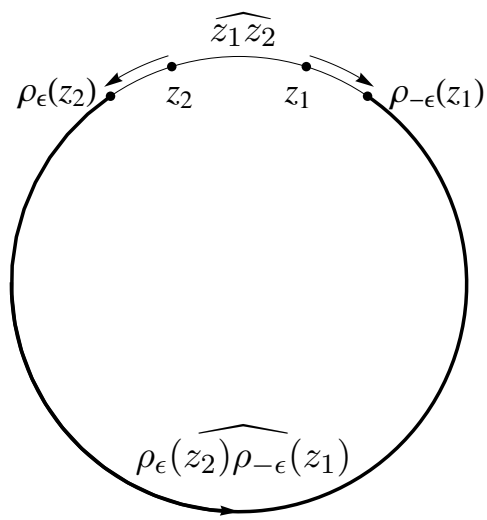

Figure 1. The points $z_{1}, z_{2}, \rho_{-\epsilon}\left(z_{1}\right), \rho_{\epsilon}\left(z_{2}\right)$ in Lemma 4 . The potential increases at every point in the subarc $\rho_{\epsilon}\left(\widehat{\left.z_{2}\right) \rho_{-\epsilon}}\left(z_{1}\right)\right.$ when $\left(z_{1}, z_{2}\right) \rightarrow\left(\rho_{-\epsilon}\left(z_{1}\right), \rho_{\epsilon}\left(z_{2}\right)\right)$; see $(9)$. 
For $\phi \in \mathbb{R}$ and $z \in \mathbb{S}^{1}$, we let $\rho_{\phi}(z):=e^{i \phi} z$ denote the counterclockwise rotation of $z$ by the angle $\phi$.

Lemma $4([2])$. Let $z_{1}, z_{2} \in \mathbb{S}^{1}$ and $0<\epsilon<\gamma\left(\widehat{z_{2} z_{1}}\right) / 2$. Then with $f$ as in Theorem 1 .

$$
U^{f}\left(\left(z_{1}, z_{2}\right) ; z\right) \leq U^{f}\left(\left(\rho_{-\epsilon}\left(z_{1}\right), \rho_{\epsilon}\left(z_{2}\right)\right) ; z\right),
$$

for $z$ in the subarc $\rho_{\epsilon}\left(\widehat{\left.z_{2}\right) \rho_{-\epsilon}}\left(z_{1}\right)\right.$, while the reverse inequality holds for $z$ in the subarc $\widehat{z_{1} z_{2}}$. If $f$ is strictly convex on $(0, \pi]$, then these inequalities are strict. If $z_{1}=z_{2}$, then we set $\widehat{z_{1} z_{2}}=\left\{z_{1}\right\}$ and $\widehat{z_{2} z_{1}}=\mathbb{S}^{1}$.

We now assume that $\omega_{n}=\left(z_{1}, \ldots, z_{n}\right)$ is ordered in a counterclockwise manner and also that the indexing is extended periodically so that $z_{k+n}=z_{k}$ for $k \in \mathbb{Z}$. For $1 \leq k \leq n$ and $\Delta \in \mathbb{R}$, we define $\tau_{k, \Delta}:\left(\mathbb{S}^{1}\right)^{n} \rightarrow\left(\mathbb{S}^{1}\right)^{n}$ by

$$
\tau_{k, \Delta}\left(z_{1}, \ldots, z_{k}, z_{k+1}, \ldots, z_{n}\right):=\left(z_{1}, \ldots, \rho_{-\Delta}\left(z_{k}\right), \rho_{\Delta}\left(z_{k+1}\right), \ldots, z_{n}\right) .
$$

If $z_{k-1} \neq z_{k}$ and $z_{k+1} \neq z_{k+2}$, then $\tau_{k, \Delta}\left(\omega_{n}\right)$ retains the ordering of $\omega_{n}$ for $\Delta$ positive and sufficiently small. Given $\boldsymbol{\Delta}:=\left(\Delta_{1}, \ldots, \Delta_{n}\right)^{T} \in \mathbb{R}^{n}$, let $\tau_{\boldsymbol{\Delta}}:=\tau_{n, \Delta_{n}} \circ \cdots \circ \tau_{2, \Delta_{2}} \circ \tau_{1, \Delta_{1}}$ and $\omega_{n}^{\prime}:=\tau_{\boldsymbol{\Delta}}\left(\omega_{n}\right)$. Letting $\alpha_{k}:=\gamma\left(\widehat{z_{k} z_{k+1}}\right)$ and $\alpha_{k}^{\prime}:=\gamma\left(\widehat{z_{k}^{\prime} z_{k+1}^{\prime}}\right)$ for $k=1, \ldots, n$, we obtain the system of $n$ linear equations:

$$
\alpha_{k}^{\prime}=\alpha_{k}-\Delta_{k-1}+2 \Delta_{k}-\Delta_{k+1}, \quad(1 \leq k \leq n),
$$

which is satisfied as long as $\sum_{k=1}^{n} \alpha_{k}^{\prime}=2 \pi$ or, equivalently, if $\omega_{n}^{\prime}$ is ordered counterclockwise. Let

$$
\operatorname{sep}\left(\omega_{n}\right):=\min _{1 \leq \ell \leq n} \alpha_{\ell}
$$

Then (10) holds if

$$
\max _{1 \leq k \leq n}\left|\Delta_{k}\right| \leq(1 / 4) \operatorname{sep}\left(\omega_{n}\right)
$$

in which case, the configurations

$$
\omega_{n, \Delta}^{(\ell)}:=\tau_{n, \Delta_{\ell}} \circ \cdots \circ \tau_{2, \Delta_{2}} \circ \tau_{1, \Delta_{1}}\left(\omega_{n}\right), \quad(\ell=1, \ldots, n)
$$

are all ordered counterclockwise. If the components of $\boldsymbol{\Delta}$ are nonnegative, then we may replace the ' $(1 / 4)$ ' in (11) with ' $(1 / 2)$ '.

Lemma 5. Suppose $\omega_{n}=\left(z_{1}, \ldots, z_{n}\right)$ and $\omega_{n}^{\prime}=\left(z_{1}^{\prime}, \ldots, z_{n}^{\prime}\right)$ are $n$-point configurations on $\mathbb{S}^{1}$ ordered in a counterclockwise manner. Then there is a unique $\boldsymbol{\Delta}^{*}=\left(\Delta_{1}^{*}, \ldots, \Delta_{n}^{*}\right) \in \mathbb{R}^{n}$ so that

(a) $\Delta_{k}^{*} \geq 0, k=1, \ldots, n$,

(b) $\Delta_{j}^{*}=0$ for some $j \in\{1, \ldots, n\}$, and

(c) $\tau_{\Delta^{*}}\left(\omega_{n}\right)$ is a rotation of $\omega_{n}^{\prime}$.

Proof. The system (10) can be expressed in the form

$$
A \Delta=\boldsymbol{\beta},
$$


where

$$
A:=\left(\begin{array}{cccccc}
2 & -1 & 0 & 0 & \cdots & -1 \\
-1 & 2 & -1 & 0 & \cdots & 0 \\
\vdots & & & & & \vdots \\
0 & 0 & \cdots & -1 & 2 & -1 \\
-1 & 0 & \cdots & 0 & -1 & 2
\end{array}\right), \quad \boldsymbol{\Delta}:=\left(\begin{array}{c}
\Delta_{1} \\
\Delta_{2} \\
\vdots \\
\Delta_{n}
\end{array}\right) \text {, and } \boldsymbol{\beta}:=\left(\begin{array}{c}
\alpha_{1}^{\prime}-\alpha_{1} \\
\alpha_{2}^{\prime}-\alpha_{2} \\
\vdots \\
\\
\alpha_{n}^{\prime}-\alpha_{n}
\end{array}\right) \text {. }
$$

It is elementary to verify that $\operatorname{ker} A=(\text { range } A)^{\perp}=\operatorname{span}(\mathbf{1})$, where $\mathbf{1}=$ $(1,1, \ldots, 1)^{T}$. Since $\boldsymbol{\beta}^{T} \mathbf{1}=\sum_{k=1}^{n}\left(\alpha_{k}^{\prime}-\alpha_{k}\right)=0$, the linear system 13. always has a solution $\boldsymbol{\Delta}$. Let $j \in\{1, \ldots, n\}$ satisfy $\Delta_{j}=\min _{1 \leq k \leq n} \Delta_{k}$. Then subtracting $\Delta_{j} \mathbf{1}$ from $\boldsymbol{\Delta}$, we obtain the desired $\boldsymbol{\Delta}^{*}$. Since ker $A=$ span $\mathbf{1}$, there is at most one solution of (13) satisfying properties (a) and (b), showing that $\boldsymbol{\Delta}^{*}$ is unique.

Part (c) holds as a direct result of the fact that both $\omega_{n}$ and $\omega_{n}^{\prime}$ are ordered counterclockwise.

Lemma 6. Let $\Omega_{n}=\left(z_{1}, \ldots, z_{n}\right)$ be a configuration of $n$ distinct points on $\mathbb{S}^{1}$ ordered counterclockwise, and with $f$ as in Theorem 1 , suppose $\boldsymbol{\Delta}=$ $\left(\Delta_{1}, \ldots, \Delta_{n}\right) \in \mathbb{R}^{n}$ is such that

(a) $0 \leq \Delta_{k} \leq(1 / 2) \operatorname{sep}\left(\Omega_{n}\right)$ for $k=1, \ldots, n$, and

(b) there is some $j \in\{1, \ldots, n\}$ for which $\Delta_{j}=0$.

Let $\Omega_{n}^{\prime}:=\tau_{\Delta}\left(\Omega_{n}\right)=\left(z_{1}^{\prime}, \ldots, z_{n}^{\prime}\right)$. Then $\widehat{z_{j}^{\prime} z_{j+1}^{\prime}} \subset \widehat{z_{j} z_{j+1}}$ and

$$
U^{f}\left(\Omega_{n} ; z\right) \leq U^{f}\left(\Omega_{n}^{\prime} ; z\right) \quad\left(z \in \widehat{z_{j}^{\prime} z_{j+1}^{\prime}}\right) .
$$

If $f$ is strictly convex on $(0, \pi]$ and $\Delta_{k}>0$ for at least one $k$, then the inequality (14) is strict.

We remark that $\Delta_{k}=0$ for all $k=1, \ldots, n$ is equivalent to saying that the points are equally spaced.

Proof. Recalling (12), it follows from condition (a) that $\left(z_{1}^{(\ell)}, \ldots, z_{n}^{(\ell)}\right):=$ $\omega_{n, \boldsymbol{\Delta}}^{(\ell)}$ are counterclockwise ordered. Since $\Delta_{j}=0$ and $\Delta_{k} \geq 0$ for $k=$ $1, \ldots, n$, the points $z_{j}^{(\ell)}$ and $z_{j+1}^{(\ell)}$ are moved at most once as $\ell$ varies from 1 to $n$ and move toward each other, while remaining in the complement of all other subarcs $z_{k}^{\widehat{(\ell)} z_{k+1}^{(\ell)}}$, i.e.,

$$
\widehat{z_{j}^{\prime} z_{j+1}^{\prime}}=\overline{z_{j}^{(n)} z_{j+1}^{(n)}} \subseteq \widehat{z_{j}^{(\ell)} z_{j+1}^{(\ell)}} \subseteq \widehat{z_{k+1}^{(\ell)} z_{k}^{(\ell)}},
$$

for $k \in\{1, \ldots, n\} \backslash\{j\}$ and $\ell \in\{1, \ldots, n\}$. Lemma 4 implies that, for $\ell=1, \ldots, n$, we have $U^{f}\left(\omega_{n}^{(\ell-1)} ; z\right) \leq U^{f}\left(\omega_{n}^{(\ell)} ; z\right)$ for $z \in \overline{z_{j}^{(\ell)} z_{j+1}^{(\ell)}}$ (where $\omega_{n}^{(0)}:=\omega_{n}$ ) and the inequality is strict if $\Delta_{\ell}>0$. Hence, (14) holds and the inequality is strict if $f$ is strictly convex and $\Delta_{k}>0$ for some $k=$ $1, \ldots, n$. 
We now proceed with the proof of Theorem 1. Let $\omega_{n}=\left(z_{1}, \ldots, z_{n}\right)$ be a non-equally spaced configuration of $n$ (not necessarily distinct) points on $\mathbb{S}^{1}$ ordered counterclockwise. By Lemma 5 , there is some equally spaced configuration $\omega_{n}^{\prime}$ (i.e., $\alpha_{k}^{\prime}=2 \pi / n$ for $\left.k=1, \ldots, n\right)$ and some $\boldsymbol{\Delta}^{*}=\left(\Delta_{1}^{*}, \ldots, \Delta_{n}^{*}\right)$ such that (a) $\omega_{n}^{\prime}=\tau_{\Delta^{*}}\left(\omega_{n}\right)$, (b) $\Delta_{k}^{*} \geq 0$ for $k=1, \ldots, n$, and (c) $\Delta_{j}^{*}=0$ for some $j \in\{1, \ldots, n\}$. Then (10) holds with $\alpha_{k}:=\gamma\left(\widehat{z_{k}, z_{k+1}}\right)$ and $\alpha_{k}^{\prime}:=2 \pi / n$. Since $\omega_{n}$ is not equally spaced, we have $\Delta_{k}^{*}>0$ for at least one value of $k$.

For $0 \leq t \leq 1$, let $\omega_{n}^{t}:=\tau_{\left(t \boldsymbol{\Delta}^{*}\right)}\left(\omega_{n}\right)=\left(z_{1}^{t}, \ldots, z_{n}^{t}\right)$ and, for $k=1, \ldots, n$, let $\alpha_{k}^{t}:=\gamma\left(\widehat{z_{k}^{t} z_{k+1}^{t}}\right)$. Recalling (10), observe that

$$
\begin{aligned}
\alpha_{k}^{t} & =\alpha_{k}-t\left(\Delta_{k-1}+2 \Delta_{k}-\Delta_{k+1}\right) \\
& =\alpha_{k}+t\left(2 \pi / n-\alpha_{k}\right) \\
& =(1-t) \alpha_{k}+t(2 \pi / n),
\end{aligned}
$$

for $0 \leq t \leq 1$ and $k=1, \ldots, n$, and $\operatorname{so~} \operatorname{sep}\left(\omega_{n}^{t}\right) \geq t(2 \pi / n)$. Now let $0<$ $t<s<\min (1, t(1+\pi /(n D)))$, where $D:=\max \left\{\Delta_{k}: 1 \leq k \leq n\right\}$. Then Lemma 6 (with $\Omega_{n}=\omega_{n}^{t}, \boldsymbol{\Delta}=(s-t) \boldsymbol{\Delta}^{*}$, and $\Omega_{n}^{\prime}=\tau_{\boldsymbol{\Delta}}\left(\Omega_{n}\right)=\omega_{n}^{s}$ ) implies that $\widehat{z_{j}^{s} z_{j+1}^{s}} \subseteq \widehat{z_{j}^{t} z_{j+1}^{t}}$ and that

$$
U^{f}\left(\omega_{n}^{t} ; z\right) \leq U^{f}\left(\omega_{n}^{s} ; z\right) \quad\left(z \in \widehat{z_{j}^{s} z_{j+1}^{s}}\right),
$$

where the inequality is sharp if $f$ is strictly convex.

Consider the function

$$
h(t):=\min \left\{U^{f}\left(\omega_{n}^{t} ; z\right): z \in \widehat{z_{j}^{t} z_{j+1}^{t}}\right\}, \quad(0 \leq t \leq 1) .
$$

Observe that

$h(t) \leq \min \left\{U^{f}\left(\omega_{n}^{t} ; z\right): z \in \widehat{z_{j}^{s} z_{j+1}^{s}}\right\} \leq \min \left\{U^{f}\left(\omega_{n}^{s} ; z\right): z \in \widehat{z_{j}^{s} z_{j+1}^{s}}\right\}=h(s)$,

for $0<t<s<\min (1, t(1+\pi /(n D)))$. It is then easy to verify that $h$ is non-decreasing on $(0,1)$. Since $\omega_{n}^{t}$ depends continuously on $t$, the function $h$ is continuous on $[0,1]$ and thus $h$ is non-decreasing on $[0,1]$.

We then obtain the desired inequality

$$
M^{f}\left(\omega_{n} ; \mathbb{S}^{1}\right) \leq h(0) \leq h(1)=M^{f}\left(\omega_{n}^{\prime} ; \mathbb{S}^{1}\right),
$$

where the last equality is a consequence of the fact that $\omega_{n}^{\prime}$ is an equally spaced configuration and so the minimum of $U^{f}\left(\omega_{n}^{\prime} ; z\right)$ over $\mathbb{S}^{1}$ is the same as the minimum over $\widehat{z_{j}^{\prime} z_{j+1}^{\prime}}$. If $f$ is strictly convex, then $h(0)<h(1)$ showing that any optimal $f$-polarization configuration must be equally spaced. This completes the proof of Theorem 1 .

Acknowledgements: We thank the referees for their helpful suggestions to improve the manuscript. 


\section{REFERENCES}

[1] G. Ambrus, Analytic and Probabilistic Problems in Discrete Geometry, Ph.D. Thesis, University College London, 2009.

[2] G. Ambrus, K. Ball, and T. Erdélyi, Chebyshev constants for the unit circle, Bull. London Math. Soc. 45(2) (2013), 236-248.

[3] J.S. Brauchart, D.P. Hardin, and E.B. Saff, The Riesz energy of the $N$ th roots of unity: an asymptotic expansion for large N, Bull. London Math. Soc., 41 (4) (2009), 621-633.

[4] J.S. Brauchart, D.P. Hardin and E.B. Saff, Discrete energy asymptotics on a Riemannian circle, Uniform Distribution Theory, 6 (2011), 77-108.

[5] T. Erdélyi and E.B. Saff, Riesz polarization inequalities in higher dimensions, (submitted), J. Approx. Theory 171 (2013), 128-147.

[6] N. Nikolov and R. Rafailov, On the sum of powered distances to certain sets of points on the circle, Pacific J. Math., 253(1), (2011), 157-168.

Center for Constructive Approximation, Department of Mathematics, VanDerbilt University, 1326 Stevenson Center, Nashville, TN, 37240, USA

E-mail address: doug.hardin@vanderbilt.edu

E-mail address: amos.p.kendall@vanderbilt.edu

E-mail address: edward.b.saff@vanderbilt.edu 